\begin{tabular}{|c|l|}
\hline Title & SPA TIO-TEMPORAL CHA OS IN A DISCRETE TURING MODEL \\
\hline Author(s) & KANG, HUNSEOK \\
\hline Citation & Hokkaido University Preprint Series in Mathematics, 931, 1-20 \\
\hline Issue Date & 2008 12-8 \\
\hline DOI & 10.14943/84079 \\
\hline Doc URL & http://hdl.handle.net/2115/69739 \\
\hline Type & bulletin (article) \\
\hline File Information & pre931.pdf \\
\hline
\end{tabular}

Instructions for use 


\title{
SPATIO-TEMPORAL CHAOS IN A DISCRETE TURING MODEL
}

\author{
HUNSEOK KANG
}

\begin{abstract}
In this paper, a discrete version of a reaction-diffusion equation, also known as coupled map lattice (CML), which corresponds to the Turing model of morphogenesis is studied. It is shown that CML possesses a hyperbolic property displaying a type of spatio-temporal chaos. Throughout a mathematical background of hyperbolic properties in lattice dynamical systems which are related to spatio-temporal chaos, a mathematical proof is given that the CML obtained from the Turing model possesses such hyperbolic properties. Finally, numerical studies of this finding in varying parameters to present a variety of chaotic behaviors of this system is performed.
\end{abstract}

\section{INTRODUCTION}

In 1952, A. Turing proposed a theoretical model for spatial pattern formation using reaction-diffusion mechanism. In his work [16] and its extensions [5, 10], it was shown that the diffusion in the mechanism may break stability in the system of reacting chemicals, and that this diffusion-driven instability results in spatially periodic patterns that are stationary in time. One of the remarkable features in this system is that the resulting pattern formation arises autonomously without referring to any external sources, such as length scales or constraints.

The morphogenesis is an embryological development of the structure of an organism or some part of an organism. Turing suggested a model of an idealized embryo which contains two characteristic chemical substances $\mathcal{X}$ and $\mathcal{Y}$ called morphogens. These substances react with each other in each cell and diffuse between neighboring cells with diffusibility coefficients $\mu$ and $\nu$ respectively. Consider an idealized embryo which is realized as an annulus of the inner radius $\rho$ of tissue (see Figure 1 ). Concentrations of the corresponding chemicals $\mathcal{X}$ and $\mathcal{Y}$ are denoted by $X$ and $Y$, respectively. Let $K_{1}(X, Y)$ and $K_{2}(X, Y)$ be kinetic functions representing the rates at which concentrations $X$ and $Y$, respectively, change due to the chemical interaction.

The reaction rates are assumed to obey the law of mass action that states the rate at which a reaction takes place is proportional to the concentrations of the reacting substances. It is also assumed that diffusion obeys the ordinary law of diffusion. In this case, each morphogen moves from the region of greater concentration to the region of lesser one at a rate proportional to the gradient of the concentration.

1991 Mathematics Subject Classification. 37N25, 37N10, 35K57.

Key words and phrases. Turing Model, Coupled Map Lattices, Traveling Wave, Hyperbolic set, Smale-horseshoe, Spatio-Temporal Chaos. 


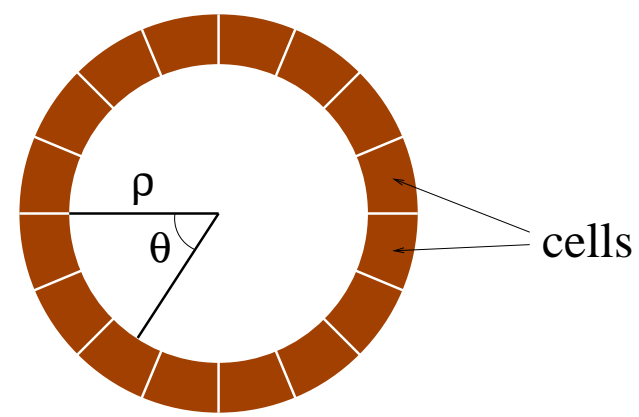

Figure 1. Turing's model of an idealized embryo.

Then the governing equations are

$$
\begin{aligned}
X_{t} & =K_{1}(X, Y)+\frac{\mu}{\rho^{2}} X_{\theta \theta}, \\
Y_{t} & =K_{2}(X, Y)+\frac{\nu}{\rho^{2}} Y_{\theta \theta},
\end{aligned}
$$

where $\theta$ is the angle between the radius to the current point and a fixed reference radius. Nonlinearity of the kinetic functions in (1.1) is an essential ingredient and is a source of the instability in the system. Turing used the following formulas for the kinetic functions

$$
\begin{aligned}
& K_{1}(X, Y)=-A X^{2}-B X Y+C, \\
& K_{2}(X, Y)=A X^{2}+B X Y-D Y+E,
\end{aligned}
$$

where $A, B, C, D, E \in \mathbb{R}^{+}$are parameters of the reaction in (1.1).

We shall discuss the dynamics of a discrete version of the Turing continuous model of the idealized embryo. When a reaction-diffusion equation is discretized, it forms a CML as usual. When discretizing Equation (1.1) the following discretizations are employed: For the time derivative in (1.1)

$$
\frac{\partial u(x, t)}{\partial t} \rightarrow \frac{u(x, t+\Delta t)-u(x, t)}{\Delta t} .
$$

And for the space derivative

$$
\frac{\partial^{2} u(x, t)}{\partial x^{2}} \rightarrow \frac{u(x+\Delta x, t)-2 u(x, t)+u(x-\Delta x, t)}{(\Delta x)^{2}} .
$$

By plugging the discretizations (1.3) and (1.4) into the system (1.1), we obtain a coupled map lattice (CML) of the type:

$$
u_{j}(n+1)=f\left(u_{j}(n)\right)+\epsilon g_{j}\left(\left\{u_{i}(n)\right\}_{|i-j| \leq s}\right),
$$

where $n \in \mathbb{Z}$ is the discrete time coordinate and $j \in \mathbb{Z}$ the discrete space coordinate. Furthermore, $f: \mathbb{R}^{2} \rightarrow \mathbb{R}^{2}$ is the local map for the CML and is given by

$$
f\left(u_{1}, u_{2}\right)=\left(u_{1}-a u_{1}^{2}-b u_{1} u_{2}+c, a u_{1}^{2}+b u_{1} u_{2}+(1-d) u_{2}+e\right),
$$

where $a=A \Delta t>0, b=B \Delta t>0, c=C \Delta t>0, d=D \Delta t>0$ and $e=E \Delta t>0$ are parameters. Finally, $g:\left(\mathbb{R}^{2}\right)^{2 s+1} \rightarrow \mathbb{R}^{2}$ is the interaction of finite size s. By selecting small discretization steps appropriately, it is assumed that $\epsilon$ is a sufficiently small parameter. 
Referred to Turing's works, a number of reaction-diffusion models have been proposed for pattern formations. These all rely on the diffusion-driven instability mechanism proposed by Turing, differing only in the terms that govern reaction kinetics $K_{1}$ and $K_{2}$ of (1.1), like in (1.2). A considerable amount of existing works on the proposed models have been mostly involved in numerical and experimental approaches to study spatio-temporal dynamics ([9], [11], [15], etc.). Throughout the study, using dynamical system approaches on the basis of mathematical definitions and proofs, we shall analyze spatio-temporal chaotic dynamics which is physically observable in a finite-dimensional phase space.

The main goal of this paper is to study the dynamics of the local map $f$ (1.6), focusing on its hyperbolic structure, and finally to show that the lattice system (1.5) displays spatio-temporal chaos. In [13], it was shown that a lattice system obtained from the discretization of the Maginu model of morphogenesis displays spatio-temporal chaos. This model is described by a two-dimensional nonlinear reaction diffusion equation in the Turing form of (1.1) with the reaction kinetic functions $K_{1}$ and $K_{2}$

$$
\begin{aligned}
& K_{1}(X, Y)=-a X(X-\theta)(X-1)-b Y, \\
& K_{2}(X, Y)=c X-d Y .
\end{aligned}
$$

This Maginu model is a simplified version of the Turing model, which is proposed by Maginu on the basis of FighHugh-Nagumo equation. (See [10] for details.) It was proven that the CML corresponding to this model displays spatio-temporal chaos by showing that the local map of the CML possesses a locally maximal hyperbolic set. This example had been the only known example of lattice system whose local map is hyperbolic. Let us emphasize that the CML corresponding to Turing model also exhibits spatio-temporal chaos as so does its simplified model.

\section{Review of Spatio-Temporal Chaos in Lattice Systems}

In this section, we describe hyperbolic properties of CMLs via traveling waves, which characterize instability of trajectories. Our goal in this section is to illustrate that for sufficiently small interactions, these properties are dominated by the hyperbolic behavior of the local map $f$ and lead to a type of spatio-temporal chaos in lattice systems.

We provide a formal mathematical description of lattice systems for one-dimensional case in terms of dynamical systems. (See [14] for general description of lattice systems for multi-dimensional cases.) We define a special norm called norm with weights. Namely, given $q_{1}>1, q_{2}>1$ and $u=\left(u_{j}\right) \in \mathbb{\bigotimes}_{\mathbb{Z}} \mathbb{R}^{d}, j \in \mathbb{Z}$,

$$
\|u\|_{q_{1}, q_{2}}=\sum_{j<0} \frac{\left|u_{j}\right|}{q_{1}^{-j}}+\sum_{j \geq 0} \frac{\left|u_{j}\right|}{q_{2}^{j}} .
$$

Then the CML (1.5) is described as the infinite-dimensional dynamical system $\left(\mathcal{M}_{q_{1}, q_{2}}, \Phi_{\epsilon}\right)$ with the underlying phase space

$$
\mathcal{M}_{q_{1}, q_{2}}=\left\{u=\left(u_{j}\right):\|u\|_{q_{1}, q_{2}}<\infty\right\}
$$

and the nonlinear evolution operator $\Phi=\Phi_{\epsilon}$

$$
(\Phi u)_{j}(n)=f\left(u_{j}(n)\right)+\epsilon g_{j}\left(\left\{u_{j}(n)\right\}_{|i-j| \leq s}\right) .
$$


It is noted that the dynamical system $\left(\mathcal{M}_{q_{1}, q_{2}}, \Phi\right)$ corresponds to solutions of the CML which satisfy the initial condition $\left(u_{j}(0)\right) \in \mathcal{M}_{q_{1}, q_{2}}$ and boundary condition $\|u\|_{q_{1}, q_{2}}<\infty$.

In [1], traveling wave solution was introduced as a class of solutions for the equation (1.5). Let $m$ and $l$ be relatively prime integers. Then a traveling wave solution of Equation (1.5) is a solution of the form:

$$
u_{j}(n)=\psi(l j+m n),
$$

where $\psi: \mathbb{Z} \rightarrow \mathbb{R}^{d}$ is a function. The numbers $m$ and $l$ determine the velocity of the wave. We assume $m>l s$, therefore, the velocity of the wave is large.

We consider the traveling wave map $F_{\epsilon}:\left(\mathbb{R}^{d}\right)^{l s+m} \rightarrow\left(\mathbb{R}^{d}\right)^{l s+m}$ given by

$$
F_{\epsilon}\left(x_{1}, \ldots, x_{l s+m}\right)=\left(x_{2}, \ldots, x_{l s+m}, f\left(x_{l s+1}\right)+\epsilon g\left(x_{p(i)}\right)_{i=-s}^{s}\right),
$$

where $p(i)=l(s+i)+1$. Then it was shown that the traveling wave map $F_{\epsilon}$ and the evolution operator $\Phi$ are conjugated.

Theorem 2.1 (see [1]). There exist $q_{1}^{(0)}>1, q_{2}^{(0)}>1$ such that for any $q_{1}>q_{1}^{(0)}$, $q_{2}>q_{2}^{(0)}$, the traveling wave map $F_{\epsilon}$ is smoothly conjugate to the evolution operator $\Phi$; in other words, there exists a smooth embedding $\chi$ of $\left(\mathbb{R}^{d}\right)^{l s+m}$ into $\mathcal{M}_{q_{1}, q_{2}}$ such that the following diagram is commutative:

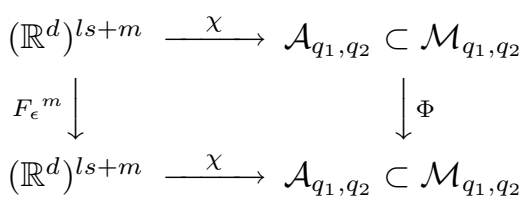

where $\mathcal{A}_{q_{1}, q_{2}}=\chi\left(\left(\mathbb{R}^{d}\right)^{l s+m}\right)$ is finite-dimensional.

Consequently, under the assumption that the interaction in (1.5) is sufficiently small, the dynamics of the evolution operator $\Phi$ on the set of traveling wave solutions with a given velocity $m / l$ is completely determined by the traveling wave $\operatorname{map} F_{\epsilon}$.

The evolution operator $\Phi$ has a special form $\Phi=F+\epsilon G$ where $F=\bigotimes_{i \in \mathbb{Z}} f_{i}$ and $G=\left(g_{j}\right)_{j \in \mathbb{Z}} . F$ is called the uncoupled map and $G$ the interaction. Iterates of the map $\Phi$ generates a $\mathbb{Z}$-action on $\mathcal{M}_{q_{1}, q_{2}}$ called time translation. On the other hand, we consider another group action of the lattice $\mathbb{Z}$ on $\mathcal{M}_{q_{1}, q_{2}}$ by space translations $S^{k}$. Namely, for any $k \in \mathbb{Z}$ and $u=\left(u_{j}\right) \in \mathcal{M}_{q_{1}, q_{2}}$, we set $\left(S^{k}(u)\right)_{i}=u_{i+k}$. If $G$ commutes with the spatial translations $S^{k}$, i.e., $S^{k} \circ G=G \circ S^{k}$, we call $G$ spatial translation invariant. In this case the pair $\tau=(\Phi, S)$ generates a $\mathbb{Z}^{2}$-action which represents a CML on $M$.

In [14], a mathematical definition of spatio-temporal chaos in lattice systems is given by Pesin and Sinai. A lattice dynamical system $(\Phi, S)$ is said to display

(1) temporal chaos if there exists a measure $\mu$ invariant under the $\mathbb{Z}$-action $\left\{\Phi^{l}\right\}$ which is mixing;

(2) spatial chaos if there exists a measure $\mu$ invariant under the $\mathbb{Z}$-action $\left\{S^{k}\right\}$ which is mixing;

(3) spatio-temporal chaos if there exists a measure $\mu$ invariant under the $\mathbb{Z}^{2}$ action $\left\{\Phi^{l}, S^{k}\right\}$ which is mixing. 
We consider the case when the local map $f$ possesses a locally maximal hyperbolic set $\Lambda$. In particular, if the local map is hyperbolic in a strong sense (i.e., it possesses a hyperbolic set; every trajectory in this set is highly unstable; see below) then so are the traveling wave map and the restrictions of space and time translations to the submanifold of traveling wave solutions. This implies that the CML displays chaotic behavior of the highest degree, i.e., there exists a measure invariant under space and time translations which is supported on the set of traveling wave solutions and has ergodic properties of higher order.

Recall that a compact invariant set $\Lambda$ is called hyperbolic if for every $x \in \Lambda$ there exists a splitting of the tangent space $T_{x} M$ at $x$ into two subspaces $E^{s}(x)$ and $E^{u}(x), T M_{\Lambda}=E^{s}(x) \oplus E^{u}(x)$ such that:

(1) the splitting is invariant under the differential $d f$, i.e.,

$$
d f\left(E^{s}(x)\right)=E^{s}(f(x)), \quad d f\left(E^{u}(x)\right)=E^{u}(f(x)) ;
$$

(2) the subspaces $E^{s}(x)$ and $E^{u}(x)$ are respectively stable and unstable for the differential $d f$, i.e.,

$$
\left|d f_{x}^{n} v\right| \leq c \lambda^{n}|v|
$$

for every $v \in E^{s}(x)$ and

$$
\left|d f_{x}^{n} v\right| \geq c^{-1} \lambda^{-n}|v|
$$

for every $v \in E^{u}(x)$, where $c>0$ and $0<\lambda<1$ are constants independent of $x$ and $v$.

A hyperbolic set $\Lambda$ is said to be locally maximal if there exists an open neighborhood $V$ of $\Lambda$ such that

$$
\Lambda=\bigcap_{n=-\infty}^{\infty} f^{n}(V) .
$$

For every point $x \in \Lambda$ one can construct local stable and unstable manifolds. We denote them by $W_{l o c}^{s}(x, f)$ and $W_{l o c}^{u}(x, f)$ respectively. The local stable manifold consists of those points $y$ whose forward trajectories follow the trajectory of $x$ within the distance $\epsilon$ for sufficiently small $\epsilon$. The local unstable manifold is characterized in a similar way by reversing the time.

One can view the map $F_{\epsilon}$ as a small perturbation of the map $F_{0}$ :

$$
F_{0}\left(x_{1}, \ldots x_{k}, \ldots, x_{n}\right)=\left(x_{2}, \ldots, x_{k+1}, \ldots, f\left(x_{k}\right)\right) .
$$

Note that the map $F_{0}$ is an endomorphism and its differential $d_{x} F_{0}$ is degenerate for every $x \in\left(\mathbb{R}^{d}\right)^{l s+m}$. Therefore, in studying topological and ergodic properties of the map $F_{\epsilon}$ for sufficiently small $\epsilon$ the classical perturbation theory may not be applied directly and may need significant modifications.

Assume that $\Lambda$ is a locally maximal hyperbolic set for the local map $f$. Consider the set $\tilde{\Lambda}_{0}=\bigotimes_{i=1}^{n} \Lambda_{i}$, where $\Lambda_{i}$ are copies of $\Lambda_{i}, i=1, \ldots, n$. We have that $F_{0}\left(\tilde{\Lambda}_{0}\right) \subset \tilde{\Lambda}_{0}$ and $\tilde{\Lambda}_{0}$ is hyperbolic.

The set $\tilde{\Lambda}_{0}$ may not survive under small perturbations of $F_{0}$ (even for local diffeomorphisms). Therefore, we introduce the set

$$
\Lambda_{0}=\bigcap_{j \geq 0} F_{0}^{j}\left(\tilde{\Lambda}_{0}\right) .
$$


It is invariant under $F_{0}$, i.e., $F_{0}\left(\Lambda_{0}\right) \subset \Lambda_{0}$. It is locally maximal and hyperbolic, and it admits the following characterization: For any $y \in \Lambda_{0}$ there exists a sequence $y_{k} \in \Lambda_{0}$ such that $y_{0}=y$ and $F_{0}\left(y_{k}\right)=y_{k+1}$ for $k \in \mathbb{Z}$.

In the case of endomorphisms for every point $x$ in a locally maximal hyperbolic set one can construct a local stable manifold. It is uniquely defined and consists of those points $y$ whose forward trajectories follow the trajectory of $x$ within the distance $\epsilon$ for sufficiently small $\epsilon$. On the other hand there are many local unstable manifolds, each corresponds to a branch of preimages of $x$. We will specify later the choice of unstable manifolds.

For the endomorphism $F_{0}$, the local stable manifold at a point $x=\left(x_{i}\right) \in \Lambda_{0}$ is given by

$$
W_{l o c}^{s}\left(x, F_{0}\right)=\prod_{i=1}^{k-1} B\left(x_{i}, \epsilon\right) \times \prod_{i=k}^{n} W_{l o c}^{s}\left(x_{i}, f\right),
$$

where $\epsilon>0$ is sufficiently small. We will define the local unstable manifold at $x$ by

$$
W_{l o c}^{u}\left(x, F_{0}\right)=\prod_{i=1}^{k-1}\left\{x_{i}\right\} \times \prod_{i=k}^{n} W_{l o c}^{u}\left(x_{i}, f\right),
$$

In [1], the authors studied hyperbolic properties of the map $F_{\epsilon}$ for sufficiently small $\epsilon$ using a modification of the classical perturbation theorem for hyperbolic sets.

Theorem 2.2 (See [1]). There exists $\epsilon_{0}>0$ such that for any $0<\epsilon<\epsilon_{0}$ there is an invariant locally maximal hyperbolic set $\Lambda_{\epsilon}$ for $F_{\epsilon}$.

By Theorem 2.2, for sufficiently small $\epsilon$ there exists a locally maximal hyperbolic set $\Lambda_{\epsilon}$ for the traveling wave map $F_{\epsilon}$. Let $\mu$ be an invariant mixing measure on $\Lambda \epsilon$. Using the map $\chi$ we can push this measure on $A_{q_{1}, q_{2}}$. Hence, we obtain the measure $\mu_{q_{1}, q_{2}}=\chi * \mu$.

Theorem 2.3 (See [1]). The measure $\mu_{q_{1}, q_{2}}$ is invariant under time and space translations and is mixing.

Therefore, the lattice dynamical system displays spatio-temporal chaos. If the local map is hyperbolic (i.e. possesses a locally maximal hyperbolic set) one can physically observe spatio-temporal chaos for the evolution operator $\Phi$ in the space traveling wave solutions.

The chaotic behavior in the infinite-dimensional phase space $\mathcal{M}_{q_{1}, q_{2}}$ associated with traveling wave solutions may not be stable in a strong sense with respect to initial data: a small change in the initial condition may cause the corresponding trajectory to diverge from the initial one. However, there is stability in a weak sense: every submanifold $\mathcal{A}_{q_{1}, q_{2}}$ (which consists of traveling wave solutions running with the velocity $m / l$ ) possesses a stable everywhere dense separatrix.

Let us note that in practice one can never deal with infinite dimensional space and therefore, cannot distinguish between metrics $\|\cdot\|_{q_{1}, q_{2}}$ with different $q_{1}$ and $q_{2}$. From this point of view the space of solutions which are bounded in space coordinate at infinity (i.e., solutions for which the norm $\|u\|=\sup _{j \in \mathbb{Z}}|u|$ ) is more practical. Let us point out that the traveling wave solutions that lie in the hyperbolic set for the evolution operator (assuming that the local map is hyperbolic) are indeed, bounded in space. More generally, any subset which is invariant under the traveling wave map and its inverse and is bounded corresponds to a set of traveling wave solutions of CML which are bounded along the space coordinate. 


\section{Hyperbolic Property}

In this section, we shall study the dynamics of the local map $f$ focusing on hyperbolic properties of the system. A detailed mathematical proof is provided for the existence of a locally maximal hyperbolic set for $f$.

3.1. Basic properties and notations. We write, for convenience, the local map $f\left(u_{1}, u_{2}\right)=\left(f_{1}\left(u_{1}, u_{2}\right), f_{2}\left(u_{1}, u_{2}\right)\right)$, where

$$
\begin{aligned}
& f_{1}\left(u_{1}, u_{2}\right)=u_{1}-a u_{1}^{2}-b u_{1} u_{2}+c, \\
& f_{2}\left(u_{1}, u_{2}\right)=a u_{1}^{2}+b u_{1} u_{2}+(1-d) u_{2}+e .
\end{aligned}
$$

The map $f$ has two fixed points for all positive values of parameters $a, b, c, d$, and $e$. We denote the fixed points by $\mathbf{q}^{+}=\left(q_{1}^{+}, q_{2}\right)$ and $\mathbf{q}^{-}=\left(q_{1}^{-}, q_{2}\right)$, where

$$
\begin{aligned}
& q_{1}^{+}=\frac{-b(c+e)+\sqrt{b^{2}(c+e)^{2}+4 a c d^{2}}}{2 a d}=\frac{2 c d}{b(c+e)+\sqrt{b^{2}(c+e)^{2}+4 a c d^{2}}} \\
& q_{1}^{-}=\frac{-b(c+e)-\sqrt{b^{2}(c+e)^{2}+4 a c d^{2}}}{2 a d}=\frac{2 c d}{b(c+e)-\sqrt{b^{2}(c+e)^{2}+4 a c d^{2}}},
\end{aligned}
$$

and $q_{2}=(c+e) / d$. The Jacobian matrix $D f_{\mathbf{p}}$ of the local map $f$ at $\mathbf{p}=\left(u_{1}, u_{2}\right)$ is

$$
D f_{\mathbf{p}}=\left[\begin{array}{rr}
1-2 a u_{1}-b u_{2} & -b u_{1} \\
2 a u_{1}+b u_{2} & b u_{1}+(1-d)
\end{array}\right] .
$$

So the characteristic function $h_{\mathbf{p}}(x)$ of $D f_{\mathbf{p}}$ is

$$
\begin{aligned}
h_{\mathbf{p}}(x) & =x^{2}-\left(2-d-2 a u_{1}+b u_{1}-b u_{2}\right) x+ \\
& +\left(1-d+b u_{1}-2 a u_{1}-b u_{2}+2 a d u_{1}+b d u_{2}\right) .
\end{aligned}
$$

Denote nullclines of $f_{1}$ and $f_{2}$ by

$$
\begin{aligned}
& N_{1}^{+}=\left\{\left(u_{1}, u_{2}\right) \mid f_{1}\left(u_{1}, u_{2}\right)=u_{1}, u_{1}>0\right\}, \\
& N_{1}^{-}=\left\{\left(u_{1}, u_{2}\right) \mid f_{1}\left(u_{1}, u_{2}\right)=u_{1}, u_{1}<0\right\}, \\
& N_{2}^{+}=\left\{\left(u_{1}, u_{2}\right) \mid f_{2}\left(u_{1}, u_{2}\right)=u_{2}, u_{2}>0\right\}, \\
& N_{2}^{-}=\left\{\left(u_{1}, u_{2}\right) \mid f_{2}\left(u_{1}, u_{2}\right)=u_{2}, u_{2}<0\right\} .
\end{aligned}
$$

The intersection of nullclines, $\left(N_{1}^{+} \cup N_{1}^{-}\right) \cap\left(N_{2}^{+} \cup N_{2}^{-}\right)$, is composed of the two fixed points, and precisely,

$$
N_{1}^{+} \cap N_{2}^{+}=\mathbf{q}^{+} \text {and } N_{1}^{-} \cap N_{2}^{+}=\mathbf{q}^{-} .
$$

Remark 3.1. The followings are obtained from direct calculations:

(1) The image of an arbitrary horizontal line in the upper half $\left(u_{1}, u_{2}\right)$-plane is an open-upward parabola whose symmetric axis has slope of -1 .

(2) The images of two arbitrary distinct horizontal lines in the upper half $\left(u_{1}, u_{2}\right)$-plane have the only one intersection.

(3) The image of a vertical line $\left\{u_{1}=k\right\}$ is a straight line represented by

$$
\left\{u_{2}=-\left(1+\frac{1-d}{b k}\right) u_{1}+\frac{1-d}{b k}\left(k-a k^{2}+c\right)+k+c+e\right\} .
$$

(4) The restriction $\left.f\right|_{\left\{u_{1}=k\right\}}$ of $f$ to a vertical line $\left\{u_{1}=k\right\}$ is one-to-one and onto the image under $f$ of $\left\{u_{1}=k\right\}$. 
3.2. Main result. A horseshoe-type construction of the local map $f$ is established to obtain a locally maximal invariant subset $\Lambda$. Then we shall show that the set $\Lambda$ possesses hyperbolic structure. As described in Section 2, the existence of the locally maximal hyperbolic set implies that the CML (1.5) displays spatio-temporal chaos.

Theorem 3.2. There exists a rectangle $\mathbf{R}=\left[s_{1}, t_{1}\right] \times\left[s_{2}, t_{2}\right] \subset \mathbb{R}^{2}$ (for some $s_{1}<q_{1}^{-}<q_{1}^{+}<t_{1}<c, 0<s_{2}<e, q_{2}<t_{2}$, and $s_{1}+t_{2}>c+e$ ) and a number $\epsilon>0$ such that for all $d$ with $|d-1|<\epsilon$ one can find $a_{0}>0$ for which if $a>a_{0}$ then

(1) the two fixed points, $\mathbf{q}^{+}=\left(q_{1}^{+}, q_{2}\right)$ and $\mathbf{q}^{-}=\left(q_{1}^{-}, q_{2}\right)$ are saddles;

(2) the set

$$
\Lambda=\bigcap_{n=-\infty}^{\infty} f^{n}(\mathbf{R})
$$

is a locally maximal hyperbolic set for $f$.

The two fixed points $\mathbf{q}^{+}$and $\mathbf{q}^{-}$are located inside the rectangle $\mathbf{R}$ in Theorem 3.2. As stated in Theorem 3.2, let the assumptions on the parameters $a$ and $d$ hold in this entire section, that is, $d$ is sufficiently close to $1, a$ is sufficiently large, and $d$ is dependent of $a$.

The constants $q_{1}^{+}$and $q_{1}^{-}$, given in (3.1), are inversely proportional to the parameter $a$. Under the conditions $s_{1}<q_{1}^{-}$and $q_{1}^{+}<t_{1}$ in Theorem 3.2, for sufficiently large $a>0, s_{1}$ and $t_{1}$ can be chosen regardless of the parameter $a$. Similarly, under the conditions that $0<s_{2}<e$ and $t_{2}>q_{2}=(c+e) / d, s_{2}$ and $t_{2}$ can be chosen regardless of $a$. Thus it is natural to assume that

Remark 3.3. The constants $s_{1}, t_{1}, s_{2}$ and $t_{2}$ do not depend on the parameter a, when $a>0$ is sufficiently large.

Denote the four corners of $\mathbf{R}$ by

$$
\mathbf{r}_{1}=\left(s_{1}, s_{2}\right), \mathbf{r}_{2}=\left(t_{1}, s_{2}\right), \mathbf{r}_{3}=\left(t_{1}, t_{2}\right) \text {, and } \mathbf{r}_{4}=\left(s_{1}, t_{2}\right),
$$

and also, four boundaries of $\mathbf{R}$ by

$$
R_{1}=\overline{\mathbf{r}_{1} \mathbf{r}_{2}}, R_{2}=\overline{\mathbf{r}_{2} \mathbf{r}_{3}}, R_{3}=\overline{\mathbf{r}_{3} \mathbf{r}_{4}} \text {, and } R_{4}=\overline{\mathbf{r}_{4} \mathbf{r}_{1}} .
$$

The corner $\mathbf{r}_{4}$ lies above the straight line $\left\{u_{1}+u_{2}=c+e\right\}$ under the assumption in Theorem 3.2. See Figure 2 for the shape of the rectangle $\mathbf{R}$.

The first statement of Theorem 3.2 is easily proven by checking the characteristic function (3.3) of the Jacobian matrix $D f_{\mathbf{p}}$ at the fixed points $\mathbf{q}^{+}$and $\mathbf{q}^{-}$. The characteristic function $h_{\mathbf{p}}$ at a point $\mathbf{p}=\left(u_{1}, u_{2}\right)$ for $x=1$ and $x=-1$ has the values

$$
\begin{aligned}
h_{\mathbf{p}}(1) & =2 a d u_{1}+b d u_{2} ; \\
h_{\mathbf{p}}(-1) & =4-2 d+2 b u_{1}-4 a u_{1}-2 b u_{2}+2 a d u_{1}+b d u_{2} .
\end{aligned}
$$

At the fixed points $\mathbf{q}^{+}$and $\mathbf{q}^{-}$,

$$
\begin{aligned}
& h_{\mathbf{q}^{+}}(1)=\sqrt{b^{2}(c+e)^{2}+4 a c d^{2}}>0 ; \\
& h_{\mathbf{q}^{-}}(1)=-\sqrt{b^{2}(c+e)^{2}+4 a c d^{2}}<0 .
\end{aligned}
$$




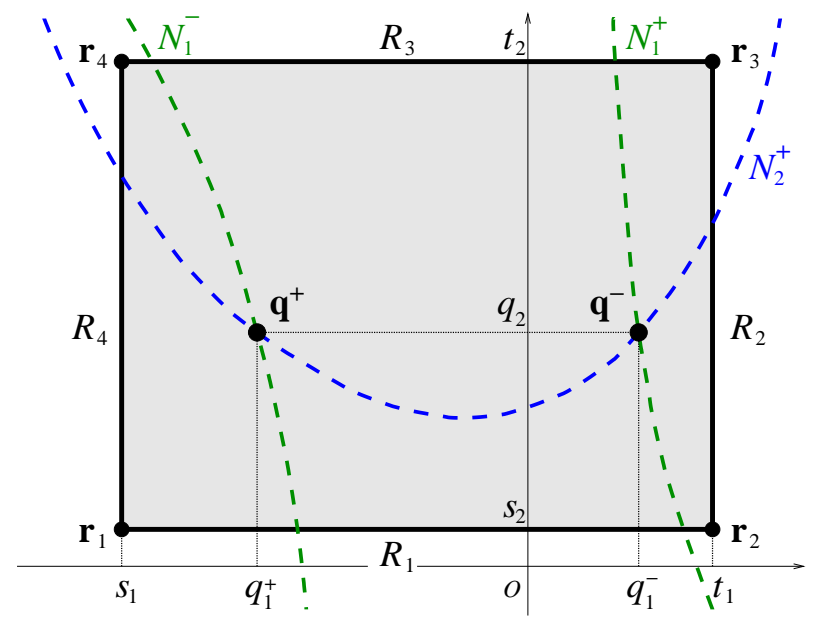

Figure 2. Bounded domain $\mathbf{R}$ in Theorem 3.2.

Thus, to show that the fixed points $\mathbf{q}^{+}$and $\mathbf{q}^{-}$are saddles, it is necessary to have the conditions $h_{\mathbf{q}^{+}}(-1)<0$ and $h_{\mathbf{q}^{-}}(-1)>0$ :

$$
\begin{aligned}
h_{\mathbf{q}^{+}}(-1) & =4-2 d+2 b q_{1}^{+}-4 a q_{1}^{+}-2 b q_{2}+2 a d q_{1}^{+}+b d q_{2} \\
& =2 b q_{1}^{+}+(2-d)\left(2-\sqrt{b^{2} q_{2}^{2}+4 a c}\right)<0, \\
h_{\mathbf{q}^{-}}(-1) & =4-2 d+2 b q_{1}^{-}-4 a q_{1}^{-}-2 b q_{2}+2 a d q_{1}^{-}+b d q_{2} \\
& =2 b q_{1}^{-}+(2-d)\left(2+\sqrt{b^{2} q_{2}^{2}+4 a c}\right)>0,
\end{aligned}
$$

for $d$ sufficiently close to 1 and sufficiently large $a$. Therefore, the two fixed points $\mathbf{q}^{+}$and $\mathbf{q}^{-}$are saddles.

In order to prove the second statement of Theorem 3.2, the following claims are proposed:

(1) $f(\mathbf{R}) \cap \mathbf{R}$ has two disjoint connected horizontal strips;

(2) $f^{-1}(\mathbf{R}) \cap \mathbf{R}$ has two disjoint connected vertical strips;

(3) $\Lambda$ is hyperbolic.

For the claims (1) and (2) above, it is necessary to understand appearance of the image $f(\mathbf{R})$ and the preimage $f^{-1}(\mathbf{R})$ around $\mathbf{R}$. We begin to study the image of the the boundaries (in Section 3.3), and then, work on the image of the interior of $\mathbf{R}$ via all the horizontal line segments in $\mathbf{R}$ and verify the claims (1) and (2) (in Section 3.4). Finally, for the claim (3), we use the cone technique to prove the hyperbolicity of $\Lambda$ (in Section 3.5).

3.3. Boundaries of $\mathbf{R}$. We study how the images of the boundaries $R_{i}, i=$ $1,2,3,4$, behave and how they are related to the others. We first check how the images of the vertical boundaries $R_{2}$ and $R_{4}$ of $\mathbf{R}$ are placed around $\mathbf{R}$.

Lemma 3.4. For $\left(u_{1}, u_{2}\right) \in R_{2} \cup R_{4}, f_{1}\left(u_{1}, u_{2}\right)<s_{1}$.

Proof. Based on Remark 3.1 (3) and (4), it suffices to show that $f_{1}\left(\mathbf{r}_{1}\right), f_{1}\left(\mathbf{r}_{2}\right)$, $f_{1}\left(\mathbf{r}_{3}\right)$, and $f_{1}\left(\mathbf{r}_{4}\right)$ are less than $s_{1}$. Since the corners $\mathbf{r}_{1}=\left(s_{1}, s_{2}\right)$ and $\mathbf{r}_{4}=\left(s_{1}, t_{2}\right)$ 
of $\mathbf{R}$ are located in the LHS of the nullcline $N_{1}^{-}$. Therefore,

$$
f_{1}\left(\mathbf{r}_{1}\right)<s_{1} \text { and } f_{1}\left(\mathbf{r}_{4}\right)<s_{1} .
$$

Hence, $f_{1}\left(u_{1}, u_{2}\right)<s_{1}$ for all $\left(u_{1}, u_{2}\right) \in R_{4}$.

On the other hand, since $s_{2}<t_{2}$,

$$
f_{1}\left(\mathbf{r}_{2}\right)=t_{1}-a t_{1}^{2}-b t_{1} s_{2}+c>t_{1}-a t_{1}^{2}-b t_{1} t_{2}+c=f_{1}\left(\mathbf{r}_{3}\right) .
$$

Since $s_{1}$ and $t_{1}$ are independent of $a$ as stated in Remark 3.3, for sufficiently large $a>0, f_{1}\left(\mathbf{r}_{2}\right)<s_{1}$, so we have

$$
f_{1}\left(\mathbf{r}_{3}\right)<f_{1}\left(\mathbf{r}_{2}\right)<s_{1} .
$$

Hence $f_{1}\left(u_{1}, u_{2}\right)<s_{1}$ for all $\left(u_{1}, u_{2}\right) \in R_{2}$.

We also check appearance of the images of the horizontal boundaries $R_{1}$ and $R_{3}$ in $\mathbf{R}$, concerning their intersections with the boundaries $R_{i}, i=1,2,3,4$.

Lemma 3.5. For $\left(u_{1}, u_{2}\right) \in R_{1} \cup R_{3}, f\left(u_{1}, u_{2}\right) \notin\left(R_{1} \cup R_{3}\right)$.

Proof. According to Remark 3.1 (1), the image of a horizontal line $\left\{u_{2}=k\right\}$, $s_{2} \leq k \leq t_{2}$, is an open-upward parabola whose symmetric axis has slope -1 . So this image has the lowest point in $\left(u_{1}, u_{2}\right)$-plane at $u_{1}=-b k /(2 a)$, which is

$$
\left(\frac{b^{2} k^{2}-2 b k}{4 a}+c,-\frac{b^{2} k^{2}}{4 a}+(1-d) k+e\right) \text {. }
$$

For sufficiently large $a,-b k /(2 a)$ is so small enough that $f\left(R_{1}\right)$ and $f\left(R_{3}\right)$ contain the lowest points in the form of (3.5) at $k=s_{2}$ and $k=t_{2}$, respectively, and since the $u_{1}$-coordinate of $(3.5)$ is

$$
t_{1}<\frac{b^{2} k^{2}-2 b k}{4 a}+c
$$

their lowest points are placed in the RHS of $R_{2}$.

Since $f_{1}(0, k)=c>t_{1}$, the image of the horizontal line $\left\{u_{2}=k\right\}$ intersects the vertical line $\left\{u_{1}=t_{1}\right\}$ at the two points

$$
\left(t_{1}, e+\left(c-t_{1}\right)+\frac{-(b k-1) \pm \sqrt{(b k-1)^{2}+4 a\left(c-t_{1}\right)}}{2 a}+(1-d) k\right) .
$$

In the $u_{2}$-coordinate of $(3.6),\left(c-t_{1}\right)$ is positive by assumption, the fraction is negligible for sufficiently large $a$, and $(1-d) k$ is negligible for $d$ sufficiently close to 1. Hence, the $u_{2}$-coordinates of the intersection (3.6) are greater than $s_{2}$.

On the other hand, by Lemma 3.4, the image of the horizontal line $\left\{u_{2}=k\right\}$ and the vertical line $\left\{u_{1}=s_{1}\right\}$ meet at two points

$$
\left(s_{1},\left(c+e-s_{1}\right)+\frac{-(b k-1) \pm \sqrt{(b k-1)^{2}+4 a\left(c-s_{1}\right)}}{2 a}+(1-d) k\right) .
$$

In the $u_{2}$-coordinate of $(3.7),(1-d) k$ and the fraction are negligible, and $\left(c+e-s_{1}\right)$ is less than $t_{2}$ by assumption in Theorem 3.2. Hence, the $u_{2}$-coordinates of the intersection (3.7) are less than $t_{2}$. Therefore, neither $f\left(R_{1}\right)$ nor $f\left(R_{3}\right)$ can touch $R_{1}$ and $R_{3}$. 


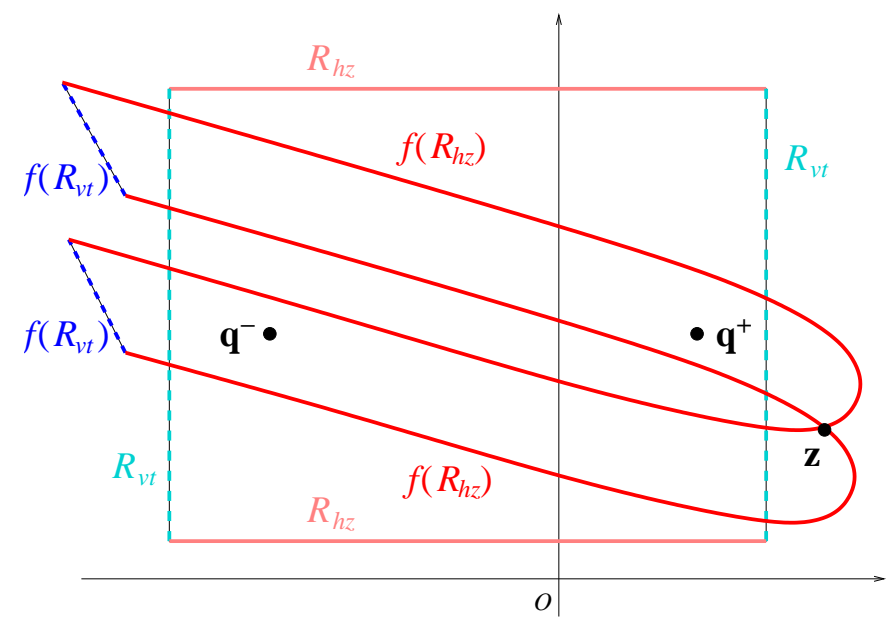

FiguRE 3. Images of boundaries of $\mathbf{R} . R_{h z}$ are the horizontal boundaries (solid lines) and $R_{v t}$ are the vertical boundaries (dotted lines). $\mathbf{z}$, given in (3.8), is the unique intersection of the images of the two horizontal boundaries.

By Lemma 3.4, all the endpoints of $f\left(R_{1}\right)$ and $f\left(R_{3}\right)$ are in the RHS of $R_{4}$. Equation (3.6) in the proof of Lemma 3.5 holds for $s_{2} \leq k \leq t_{2}$, so, by having $u_{2}=s_{2}$ and $u_{2}=t_{2}$, each of $f\left(R_{1}\right)$ and $f\left(R_{3}\right)$ has two intersections with $R_{2}$. Similarly, Equation (3.7) holds for $s_{2} \leq k \leq t_{2}$, so each of $f\left(R_{1}\right)$ and $f\left(R_{3}\right)$ also has two intersections with $R_{4}$. Thus we let

$$
f\left(R_{1}\right) \cap R_{2}=\left\{\left(t_{1}, \alpha\right),\left(t_{1}, \hat{\alpha}\right)\right\}, \quad \text { and } \quad f\left(R_{3}\right) \cap R_{2}=\left\{\left(t_{1}, \beta\right),\left(t_{1}, \hat{\beta}\right)\right\},
$$

where $\alpha>\hat{\alpha}$ and $\beta>\hat{\beta}$. The following lemma shows us how these intersections are arranged in $R_{2}$.

Lemma 3.6. Either $\alpha>\beta>\hat{\alpha}>\hat{\beta}$ or $\beta>\alpha>\hat{\beta}>\hat{\alpha}$.

Proof. By Remark 3.1 (2), the image of the horizontal line $\left\{u_{2}=s_{2}\right\}$ and that of $\left\{u_{2}=t_{2}\right\}$ have the only one intersection. Let $\mathbf{z}=\left(z_{1}, z_{2}\right)$ be their intersection. Then, solving the equations of $\mathbf{z}$

$$
\mathbf{z}=f\left(\zeta, s_{2}\right), \text { and } \mathbf{z}=f\left(\tilde{\zeta}, t_{2}\right)
$$

for $\zeta$ and $\tilde{\zeta}$, we have

$$
\begin{aligned}
& \zeta=\frac{(1-d)\left(1-b-(1-d)\left(s_{2}-t_{2}\right)\right)}{2 a(1-d)-b}, \\
& \tilde{\zeta}=\frac{(1-d)\left(1-b+((2 a-1)(1-d)-b)\left(s_{2}-t_{2}\right)\right)}{2 a(1-d)-b} .
\end{aligned}
$$

Both $\zeta$ and $\tilde{\zeta}$ vanish for $d$ sufficiently close to 1 , so that $\left(\zeta, s_{2}\right) \in R_{1}$ and $\left(\tilde{\zeta}, t_{2}\right) \in R_{3}$, and hence, $f\left(R_{1}\right)$ and $f\left(R_{3}\right)$ also intersect at $\mathbf{z}$. Note that

$$
z_{1}-t_{1}=f_{1}\left(\zeta, s_{2}\right)-t_{1}=\left(\zeta-a \zeta^{2}-b s_{2} \zeta+c\right)-t_{1}=\left(c-t_{1}\right)+\left(1-b s_{2}-a \zeta\right) \zeta \text {. }
$$

For $d$ sufficiently close to $1, \zeta$ is so small enough that $\left(z_{1}-t_{1}\right)>0$, because $\left(c-t_{1}\right)>0$. Therefore, the intersection $\mathbf{z}$ lies in the RHS of the boundary $R_{2}$. 


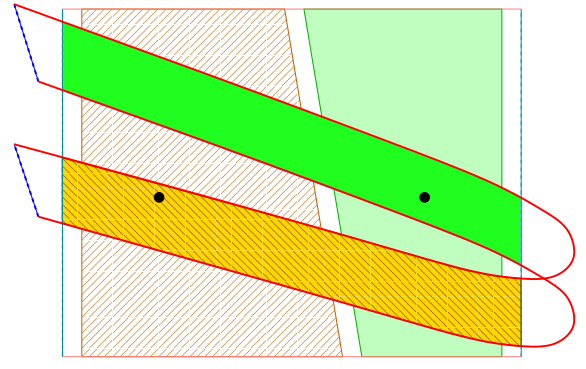

(a) $f(\mathbf{R}) \cap \mathbf{R}$.

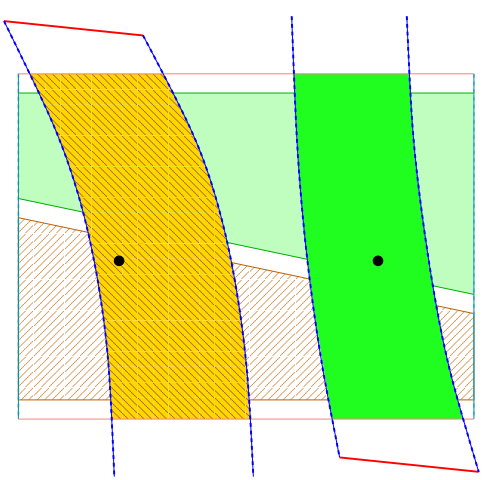

(b) $f^{-1}(\mathbf{R}) \cap \mathbf{R}$.

Figure 4. These figures illustrate that $f(\mathbf{R}) \cap \mathbf{R}$ and $f^{-1}(\mathbf{R}) \cap \mathbf{R}$ make two disjoint horizontal components and two disjoint vertical components, respectively.

From each lemma in this section, we obtain the following properties:

(1) the images of horizontal boundaries $R_{1}$ and $R_{3}$ do not meet the horizontal boundaries (See Lemma 3.5).

(2) the intersections of $f\left(R_{1}\right)$ with $R_{2}$ and those of $f\left(R_{3}\right)$ with $R_{2}$ appear alternately on $R_{2}$ (See Lemma 3.6).

(3) the images of vertical boundaries $R_{2}$ and $R_{4}$ lie in the LHS of $R_{4}$ (See Lemma 3.4).

Combining these three properties, one can see how the images of the boundaries of $\mathbf{R}$ behave, and especially, how they are related to their original preimages. See Figure 3.

3.4. Interiors of $\mathbf{R}$. As seen in Section 3.3, the images of the boundaries of $\mathbf{R}$ surround two disjoint bounded regions inside $\mathbf{R}$. See Figure 3). Let $\mathbf{R}^{+}$be one bounded region containing $\mathbf{q}^{+}$, and $\mathbf{R}^{-}$the other bounded region containing $\mathbf{q}^{-}$. Then

$$
\mathbf{R}^{+} \subset f(\mathbf{R}) \cap \mathbf{R}, \quad \text { and } \quad \mathbf{R}^{-} \subset f(\mathbf{R}) \cap \mathbf{R} .
$$

Let $\mathbf{S}^{+}$and $\mathbf{S}^{-}$be the maximal connected components in $f(\mathbf{R}) \cap \mathbf{R}$ containing $\mathbf{R}^{+}$ and $\mathbf{R}^{-}$, respectively. We shall show that $\mathbf{S}^{+}$and $\mathbf{S}^{-}$are disjoint, and hence, they are the two horizontal disjoint strips of $f(\mathbf{R}) \cap \mathbf{R}$.

Denote by $L_{\mathbf{z}}$ the line with slope of -1 passing through the point $\mathbf{z}=\left(z_{1}, z_{2}\right)$, where $\mathbf{z}$, given in the proof of Lemma 3.6, is the intersection of the images of the horizontal lines $\left\{u_{2}=s_{2}\right\}$ and $\left\{u_{2}=t_{2}\right\}$. Then $\mathbf{R}^{+}$is placed above $L_{\mathbf{z}}$ while $\mathbf{R}^{-}$is placed below $L_{\mathbf{z}}$. In Lemma 3.7, we shall show that $L_{\mathbf{z}}$ is placed between $\mathbf{S}^{+}$and $\mathbf{S}^{-}$, having no intersections with them.

On the other hand, we denote by $H_{k}$ the horizontal line segment in $\mathbf{R}$ with $u_{2}=k$, for $s_{2}<k<t_{2}$. Referred to Remark $3.1(1), f\left(H_{k}\right)$ is a part of the openupward parabola whose symmetric axis has the slope -1 . One can use the same argument as in the proof of Lemma 3.5 to show that $f\left(H_{k}\right) \cap\left(R_{1} \cup R_{3}\right)=\emptyset$ and that $f\left(H_{k}\right)$ has two intersections with $R_{2}$, and also, with $R_{4}$. 


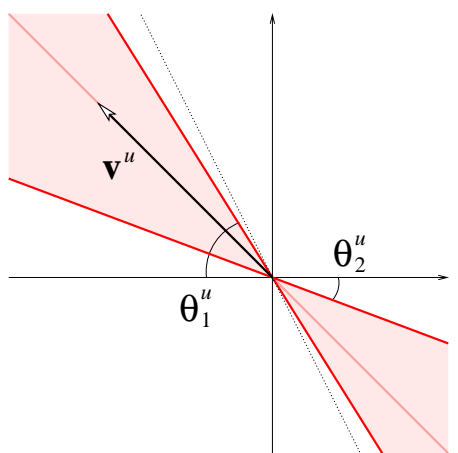

(a) Unstable cone

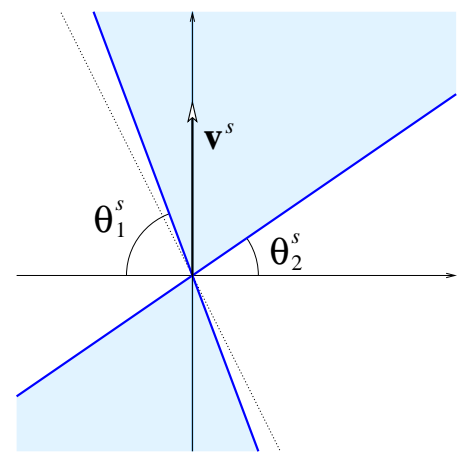

(b) Stable cone

Figure 5. The unstable and stable cones $C^{u}(\mathbf{p})$ and $C^{s}(\mathbf{p})$ in (3.10). The reference angles of the cones are $\theta_{1}^{u}=\tan ^{-1}\left(1+\left|\lambda^{-1}\right|\right)$ and $\theta_{2}^{u}=\tan ^{-1}\left(1-\left|\lambda^{-1}\right|\right)$ in (a), and $\theta_{1}^{s}=\tan ^{-1}(|\lambda|+1)$ and $\theta_{2}^{s}=\tan ^{-1}(|\lambda|-1)$ in (b).

Lemma 3.7. $L_{\mathbf{z}}$ and $f\left(H_{k}\right)$ have the only one intersection, say $\mathbf{x}=\left(x_{1}, x_{2}\right)$, and the intersection $\mathbf{x}$ lies in the RHS of $R_{2}$, i.e., $x_{1}>t_{1}$.

Proof. Since the symmetric axis of $f\left(H_{k}\right)$ has the same slope as $L_{\mathbf{z}}$, they have the only one intersection. From the equation $f_{1}\left(u_{1}, k\right)+f_{2}\left(u_{1}, k\right)=z_{1}+z_{2}$, we obtain

$$
u_{1}=(1-d)\left(z_{1}+z_{2}-k\right) .
$$

This implies that $f\left(H_{k}\right)$ intersects $L_{\mathbf{z}}$ when $u_{1}=(1-d)\left(z_{1}+z_{2}-k\right)$, so the $u_{1}$-coordinate of the intersection $\mathbf{x}$ is

$$
\begin{aligned}
x_{1} & =f_{1}\left((1-d)\left(z_{1}+z_{2}-k\right), k\right) \\
& =c+(1-d)\left(z_{1}+z_{2}-k\right)\left(1-b k-a(1-d)\left(z_{1}+z_{2}-k\right)\right) .
\end{aligned}
$$

Therefore, for $d$ sufficiently close to $1,\left(x_{1}-c\right)$ vanishes so that $x_{1}>t_{1}$.

By Lemma 3.7, the line $L_{\mathbf{z}}$ is placed between $\mathbf{S}^{+}$and $\mathbf{S}^{-}$without intersecting them. Therefore, $f(\mathbf{R}) \cap \mathbf{R}$ is composed of two disjoint connected horizontal strips $\mathbf{S}^{+}$and $\mathbf{S}^{-}$. Using the same method, one can show that $f^{-1}(\mathbf{R}) \cap \mathbf{R}$ is composed of two disjoint connected vertical strips. See Figure 4.

3.5. Hyperbolicity of $\Lambda$. We define the cone fields $C^{u}(\mathbf{p})$ and $C^{s}(\mathbf{p})$ by

$$
\begin{aligned}
& C^{u}(\mathbf{p})=\left\{\mathbf{v}=\xi \mathbf{v}^{u}+\eta \mathbf{v}^{s} \in T_{\mathbf{p}} \mathbb{R}^{2}:|\eta| \leq \lambda^{-1}|\xi|\right\}, \\
& C^{s}(\mathbf{p})=\left\{\mathbf{v}=\xi \mathbf{v}^{u}+\eta \mathbf{v}^{s} \in T_{\mathbf{p}} \mathbb{R}^{2}:|\eta| \geq \lambda|\xi|\right\},
\end{aligned}
$$

where $\mathbf{v}^{u}=(-1,1)$ and $\mathbf{v}^{s}=(0,1)$. Then for given $\xi>0$ and $\eta>0, \mathbf{v}=(-\xi, \xi+\eta)$. Figure 5 shows us the shape of the cones $C^{u}(\mathbf{p})$ and $C^{s}(\mathbf{p})$. For ease in estimation, we use the norm which measures the larger component of a vector: $\left\|\left(v_{1}, v_{2}\right)\right\|=$ $\max \left\{\left|v_{1}\right|,\left|v_{2}\right|\right\}$. We find a $\lambda>1$ that makes the cones strictly invariant.

This method showing a given invariant set is hyperbolic is called the cone technique, which is introduced in [2] and constantly developed as in [4], [12], etc. Especially, in [4], the cone criterion was applied to show that the hénon map has hyperbolic sets. 
Proposition 3.8. There is a $\lambda>1$ for which the following two statements are true.

(1) For all $\mathbf{p} \in \mathbf{R} \cap f^{-1}(\mathbf{R})$ and $\mathbf{v} \in C^{u}(\mathbf{p}), D f_{\mathbf{p}} \mathbf{v} \in C^{u}(f(\mathbf{p}))$ and $\left\|D f_{\mathbf{p}} \mathbf{v}\right\| \geq$ $\lambda\|\mathbf{v}\|$.

(2) For all $\mathbf{p} \in \mathbf{R} \cap f(\mathbf{R})$ and $\mathbf{v} \in C^{s}(\mathbf{p}), D f_{\mathbf{p}}^{-1} \mathbf{v} \in C^{s}\left(f^{-1}(\mathbf{p})\right)$ and $\left\|D f_{\mathbf{p}}^{-1} \mathbf{v}\right\| \geq$ $\lambda\|\mathbf{v}\|$.

In order to prove Proposition 3.8, we need some estimates which we prove first in the following lemma.

Lemma 3.9. For any point $\left(u_{1}, u_{2}\right) \in f^{-1}(\mathbf{R})$,

$$
\max \left\{\left|1-2 a u_{1}-b u_{2}\right|,\left|2 a u_{1}+b u_{2}\right|\right\} \geq \sqrt{\left(b u_{2}-1\right)^{2}+2 a c} .
$$

Proof. Since $f\left(u_{1}, u_{2}\right) \in \mathbf{R}, f_{1}\left(u_{1}, u_{2}\right) \leq c / 2$. This inequality implies that one of the following inequalities holds:

$$
\begin{aligned}
& u_{1} \leq \frac{-\left(b u_{2}-1\right)-\sqrt{\left(b u_{2}-1\right)^{2}+2 a c}}{2 a}, \\
& u_{1} \geq \frac{-\left(b u_{2}-1\right)+\sqrt{\left(b u_{2}-1\right)^{2}+2 a c}}{2 a} .
\end{aligned}
$$

If (3.11) holds, then $\left|1-2 a u_{1}-b u_{2}\right|>\left|2 a u_{1}+b u_{2}\right|$ so that

$$
\begin{aligned}
\left|1-2 a u_{1}-b u_{2}\right| & \geq\left|1-2 a \cdot \frac{-\left(b u_{2}-1\right)-\sqrt{\left(b u_{2}-1\right)^{2}+2 a c}}{2 a}-b u_{2}\right| \\
& =\sqrt{\left(b u_{2}-1\right)^{2}+2 a c} .
\end{aligned}
$$

If (3.12) holds, then $\left|1-2 a u_{1}-b u_{2}\right|<\left|2 a u_{1}+b u_{2}\right|$ so that

$$
\begin{aligned}
\left|2 a u_{1}+b u_{2}\right| & \geq\left|2 a \cdot \frac{-\left(b u_{2}-1\right)+\sqrt{\left(b u_{2}-1\right)^{2}+2 a c}}{2 a}+b u_{2}\right| \\
& =1+\sqrt{\left(b u_{2}-1\right)^{2}+2 a c} .
\end{aligned}
$$

The set $\Lambda$, given in Theorem 3.2, can be replaced in the following way:

$$
\Lambda=\bigcap_{n=-\infty}^{\infty} f^{n}\left(f^{-1}(\mathbf{R})\right)\left(=\bigcap_{n=-\infty}^{\infty} f^{n}(\mathbf{R})\right) .
$$

Therefore, without loss of generality, we assume that every point $\left(u_{1}, u_{2}\right) \in \mathbf{R}$ satisfies the inequalities (3.11) and (3.12), and furthermore, $\left(u_{1}, u_{2}\right)$ has the same property as in Lemma 3.9.

Proof of Proposition 3.8. Take $\mathbf{p}=\left(u_{1}, u_{2}\right)$ with $\mathbf{p}, f(\mathbf{p}) \in \mathbf{R}$. Let $\mathbf{v}=\mathbf{v}^{u}+\mathbf{v}^{s} \in$ $C^{u}(\mathbf{p})$ where $\mathbf{v}^{u}=\xi(-1,1)$ and $\mathbf{v}^{s}=\eta(0,1)$. Then $\left\|\mathbf{v}^{u}\right\| \geq \lambda\left\|\mathbf{v}^{s}\right\|$ so that $|\xi| \geq \lambda|\eta|$, and hence, $|\xi| \geq|\eta|$.

Let $\mathbf{w}=\mathbf{w}^{u}+\mathbf{w}^{s}=D f_{\mathbf{p}}(\mathbf{v})$, where

$$
\mathbf{w}^{u}=\xi_{1}(-1,1) \quad \text { and } \quad \mathbf{w}^{s}=\eta_{1}(0,1) .
$$

Note that

$\mathbf{v}=\left[\begin{array}{rr}-1 & 0 \\ 1 & 1\end{array}\right]\left[\begin{array}{l}\xi \\ \eta\end{array}\right], \mathbf{w}=\left[\begin{array}{rr}-1 & 0 \\ 1 & 1\end{array}\right]\left[\begin{array}{l}\xi_{1} \\ \eta_{1}\end{array}\right]$, and $\left[\begin{array}{rr}-1 & 0 \\ 1 & 1\end{array}\right]^{-1}=\left[\begin{array}{rr}-1 & 0 \\ 1 & 1\end{array}\right]$. 
So we have

$$
\begin{aligned}
{\left[\begin{array}{l}
\xi_{1} \\
\eta_{1}
\end{array}\right] } & =\left[\begin{array}{rr}
-1 & 0 \\
1 & 1
\end{array}\right]\left[\begin{array}{rr}
1-2 a u_{1}-b u_{2} & -b u_{1} \\
2 a u_{1}+b u_{2} & b u_{1}+1-d
\end{array}\right]\left[\begin{array}{rr}
-1 & 0 \\
1 & 1
\end{array}\right]\left[\begin{array}{l}
\xi \\
\eta
\end{array}\right] \\
& =\left[\begin{array}{cc}
1-2 a u_{1}-b u_{2}+b u_{1} & b u_{1} \\
-d & 1-d
\end{array}\right]\left[\begin{array}{l}
\xi \\
\eta
\end{array}\right] \\
& =\left[\begin{array}{c}
\left(1-2 a u_{1}-b u_{2}+b u_{1}\right) \xi+b u_{1} \eta \\
-d \xi+(1-d) \eta
\end{array}\right] .
\end{aligned}
$$

Thus, $\left|\eta_{1}\right|=|-d \xi+(1-d) \eta| \leq(|d|+|1-d|)|\xi|$. Also,

$$
\begin{aligned}
\left|\xi_{1}\right| & =\left|\left(1-2 a u_{1}-b u_{2}+b u_{1}\right) \xi+b u_{1} \eta\right| \\
& \geq\left|1-2 a u_{1}-b u_{2}+b u_{1}\right||\xi|-\left|b u_{1}\right||\eta| \geq\left(\left|1-2 a u_{1}-b u_{2}\right|-2\left|b u_{1}\right|\right)|\xi| \\
& \geq \frac{\left|2 a u_{1}+b u_{2}-1\right|-2\left|b u_{1}\right|}{|d|+|1-d|}\left|\eta_{1}\right| \geq \frac{\sqrt{\left(b u_{2}-1\right)^{2}+2 a c}-2\left|b u_{1}\right|}{|d|+|1-d|}\left|\eta_{1}\right| .
\end{aligned}
$$

Let $\left(w_{1}, w_{2}\right)=D f_{\mathbf{p}}(\mathbf{v})$, i.e.,

$$
\left[\begin{array}{l}
w_{1} \\
w_{2}
\end{array}\right]=\left[\begin{array}{c}
\left(-1+2 a u_{1}+b u_{2}-b u_{1}\right) \xi-b u_{1} \eta \\
\left(-2 a u_{1}-b u_{2}+b u_{1}+1-d\right) \xi+\left(b u_{1}+1-d\right) \eta
\end{array}\right]
$$

Then

$$
\begin{aligned}
& \left|w_{1}\right| \geq\left(\left|2 a u_{1}+b u_{2}-1\right|-2\left|b u_{1}\right|\right)|\xi|, \\
& \left|w_{2}\right| \geq\left(\left|2 a u_{1}+b u_{2}\right|-2\left|b u_{1}\right|-|1-d|\right)|\xi| .
\end{aligned}
$$

Since $\|\mathbf{v}\|=\max \{|\xi|,|\xi+\eta|\} \leq 2|\xi|$,

$$
\begin{aligned}
\left\|D f_{\mathbf{p}}(\mathbf{v})\right\| & =\max \left\{\left|w_{1}\right|,\left|w_{2}\right|\right\} \\
& \geq\left(\sqrt{\left(b u_{2}-1\right)^{2}+2 a c}-2\left|b u_{1}\right|-|1-d|\right)|\xi| \\
& \geq \frac{\sqrt{\left(b u_{2}-1\right)^{2}+2 a c}-2\left|b u_{1}\right|-|1-d|}{2}\|\mathbf{v}\| .
\end{aligned}
$$

Therefore, we take

$$
\lambda_{1}=\min \left\{\frac{\sqrt{\left(b u_{2}-1\right)^{2}+2 a c}-2\left|b u_{1}\right|}{|d|+|1-d|}, \frac{\sqrt{\left(b u_{2}-1\right)^{2}+2 a c}-2\left|b u_{1}\right|-|1-d|}{2}\right\},
$$

so that $\left\|\mathbf{w}^{u}\right\| \geq \lambda_{1}\left\|\mathbf{w}^{s}\right\|$ and $\left\|D f_{\mathbf{p}}(\mathbf{v})\right\| \geq \lambda_{1}\|\mathbf{v}\|$. Finally, under the assumptions on $a$ and $d, \lambda_{1}>1$. This proves the first part of Proposition 3.8.

For the second part, take $\mathbf{p}=\left(u_{1}, u_{2}\right)$ with $\mathbf{p}, f^{-1}(\mathbf{p}) \in \mathbf{R}$. Let $\mathbf{v}=\mathbf{v}^{u}+\mathbf{v}^{s} \in$ $C^{s}(\mathbf{p})$ where $\mathbf{v}^{u}=\xi(-1,1)$ and $\mathbf{v}^{s}=\eta(0,1)$. Since $\left\|\mathbf{v}^{s}\right\| \geq \lambda\left\|\mathbf{v}^{u}\right\|,|\eta| \geq \lambda|\xi|$, and hence, $|\eta| \geq|\xi|$.

Let $\mathbf{w}=\mathbf{w}^{u}+\mathbf{w}^{s}=D f_{\mathbf{p}}^{-1}(\mathbf{v})$, where

$$
\mathbf{w}^{u}=\xi_{-1}(-1,1) \quad \text { and } \quad \mathbf{w}^{s}=\eta_{-1}(0,1) .
$$

Then we have

$$
\begin{aligned}
{\left[\begin{array}{c}
\xi_{-1} \\
\eta_{-1}
\end{array}\right] } & =\frac{1}{m}\left[\begin{array}{rr}
-1 & 0 \\
1 & 1
\end{array}\right]\left[\begin{array}{rr}
b u_{1}+1-d & b u_{1} \\
-2 a u_{1}-b u_{2} & 1-2 a u_{1}-b u_{2}
\end{array}\right]\left[\begin{array}{rr}
-1 & 0 \\
1 & 1
\end{array}\right]\left[\begin{array}{l}
\xi \\
\eta
\end{array}\right] \\
& =\frac{1}{m}\left[\begin{array}{rr}
1-d & -b u_{1} \\
d & 1-2 a u_{1}-b u_{2}+b u_{1}
\end{array}\right]\left[\begin{array}{l}
\xi \\
\eta
\end{array}\right] \\
& =\frac{1}{m}\left[\begin{array}{c}
d \xi+(1-2 a) \xi-b u_{1} \eta \\
\left.d \xi+b u_{2}+b u_{1}\right) \eta
\end{array}\right]
\end{aligned}
$$


where $m=m\left(u_{1}, u_{2}\right)=(1-d)\left(1-2 a u_{1}-b u_{2}\right)+b u_{1}$. Then,

$$
\left|\xi_{-1}\right|=\frac{\left|(1-d) \xi-b u_{1} \eta\right|}{\left|m\left(u_{1}, u_{2}\right)\right|} \leq \frac{\left|b u_{1}\right|+|1-d|}{\left|m\left(u_{1}, u_{2}\right)\right|}|\eta|
$$

So, we have

$$
\begin{aligned}
& \left|\eta_{-1}\right|=\frac{\left|d \xi+\left(1-2 a u_{1}-b u_{2}+b u_{1}\right) \eta\right|}{\left|m\left(u_{1}, u_{2}\right)\right|} \\
& \geq \frac{\left|1-2 a u_{1}-b u_{2}+b u_{1}\right||\eta|-|d||\xi|}{\left|m\left(u_{1}, u_{2}\right)\right|} \\
& \geq \frac{\left|1-2 a u_{1}-b u_{2}\right|-\left|b u_{1}\right|-|d|}{\left|m\left(u_{1}, u_{2}\right)\right|}|\eta| \\
& \geq \frac{\left|1-2 a u_{1}-b u_{2}\right|-\left|b u_{1}\right|-|d|}{\left|b u_{1}\right|+|1-d|}\left|\xi_{-1}\right| \\
& \geq \frac{\sqrt{\left(b u_{2}-1\right)^{2}+2 a c}-\left|b u_{1}\right|-|d|}{\left|b u_{1}\right|+|1-d|}\left|\xi_{-1}\right| .
\end{aligned}
$$

Let $\left(w_{1}, w_{2}\right)=D f_{\mathbf{p}}^{-1}(\mathbf{v})$, i.e.,

$$
\left[\begin{array}{l}
w_{1} \\
w_{2}
\end{array}\right]=\frac{1}{m}\left[\begin{array}{c}
(-1+d) \xi-b u_{1} \eta \\
\xi+\left(1-2 a u_{1}-b u_{2}\right) \eta
\end{array}\right]
$$

Then

$$
\begin{aligned}
& \left|w_{1}\right| \geq \frac{\left|b u_{1}\right|-|1-d|}{|1-d|\left|1-2 a u_{1}-b u_{2}\right|+\left|b u_{1}\right|}|\eta|, \\
& \left|w_{2}\right| \geq \frac{\left|1-2 a u_{1}-b u_{2}\right|-1}{|1-d|\left|1-2 a u_{1}-b u_{2}\right|+\left|b u_{1}\right|}|\eta| .
\end{aligned}
$$

For a sufficiently large value of $a,\left|w_{1}\right|<\left|w_{2}\right|$. Since $\|\mathbf{v}\|=\max \{|\xi|,|\xi+\eta|\} \leq 2|\eta|$,

$$
\begin{aligned}
\left\|D f_{\mathbf{p}}^{-1}(\mathbf{v})\right\| & =\max \left\{\left|w_{1}\right|,\left|w_{2}\right|\right\} \\
& \geq \frac{\left|1-2 a u_{1}-b u_{2}\right|-1}{|1-d|\left|1-2 a u_{1}-b u_{2}\right|+\left|b u_{1}\right|}|\eta| \\
& \geq \frac{\sqrt{\left(b u_{2}-1\right)^{2}+2 a c}-1}{2|1-d|\left|1-2 a u_{1}-b u_{2}\right|+2\left|b u_{1}\right|}\|\mathbf{v}\| .
\end{aligned}
$$

Therefore, we take

$$
\lambda_{2}=\min \left\{\frac{\sqrt{\left(b u_{2}-1\right)^{2}+2 a c}-\left|b u_{1}\right|-|d|}{\left|b u_{1}\right|+|1-d|}, \frac{\sqrt{\left(b u_{2}-1\right)^{2}+2 a c}-1}{2|1-d|\left|1-2 a u_{1}-b u_{2}\right|+2\left|b u_{1}\right|}\right\},
$$

so that $\left\|\mathbf{w}^{s}\right\| \geq \lambda_{2}\left\|\mathbf{w}^{u}\right\|$ and $\left\|D f_{\mathbf{p}}^{-1}(\mathbf{v})\right\| \geq \lambda_{2}\|\mathbf{v}\|$. Finally, due to the assumptions on $a$ and $d, \lambda_{2}>1$. This completes the proof of Proposition 3.8.

\section{Numerical Results}

In the section, we shall provide examples of hyperbolic sets of the local map $f$. Referring to the conditions on the parameters in Theorem 3.2, we build a horseshoetype construction for $f$ to setup the invariant set $\Lambda$ in Theorem 3.2, and then search for a proper constant $\lambda$ given in (3.10) which makes the two cone fields in (3.10) strictly invariant so that the set $\Lambda$ is hyperbolic. 


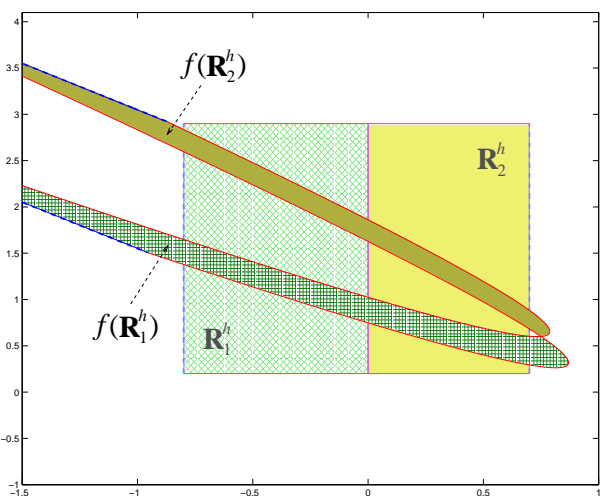

(a) $f\left(\mathbf{R}_{1}^{h}\right)$ and $f\left(\mathbf{R}_{2}^{h}\right)$.

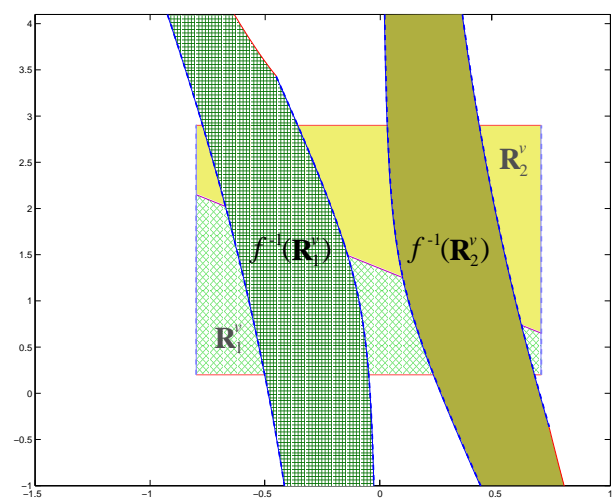

(b) $f^{-1}\left(\mathbf{R}_{1}^{v}\right)$ and $f^{-1}\left(\mathbf{R}_{2}^{v}\right)$.

Figure 6 . At $a=4.5, b=0.85, c=0.75, d=1$, and $e=0.6$, the two disjoint horizontal strips and the two disjoint vertical strips which constitute a Smale-horseshoe. In (a), the line between $\mathbf{R}_{1}^{h}$ and $\mathbf{R}_{2}^{h}$ is mapped to the intersection of $f\left(\mathbf{R}_{1}^{h}\right)$ and $f\left(\mathbf{R}_{2}^{h}\right)$. In (b), however, the preimage of the line between $\mathbf{R}_{1}^{v}$ and $\mathbf{R}_{2}^{v}$ does not exist in the plane, and it may be viewed as the intersection of $f^{-1}\left(\mathbf{R}_{1}^{v}\right)$ and $f^{-1}\left(\mathbf{R}_{2}^{v}\right)$, which is the infinity.

In Section 3, it was revealed that the existence of hyperbolic sets of the local map $f(1.6)$ is mainly dependent of the values of the parameters $a$ and $d$. We deal with two cases when $d=1$, and when $d$ is not equal to 1 but as close to 1 as necessary, while letting $a$ be large enough.

4.1. $d=1$. One can have easily comprehensible examples for hyperbolic sets when $d=1$. We consider the image under $f$ of an arbitrary vertical line $\left\{u_{1}=k\right\}$ in $\mathbb{R}^{2}$. When $u_{1}=k=0$, since $f\left(0, u_{2}\right)=(c, e)$ for all $u_{2} \in \mathbb{R}$, the image of the vertical line $\left\{u_{1}=k\right\}$ is a point $(c, e)$. So, by Remark $3.1(2)$, the images of two arbitrary distinct horizontal lines meet only at the point $(c, e)$.

On the other hand, when $u_{1}=k \neq 0$, the image of the vertical line $\left\{u_{1}=k\right\}$ is the straight line $\left\{u_{1}+u_{2}=k+c+e\right\}$ (by plugging $d=1$ into (3.4)), so that the images of two arbitrary distinct vertical lines do not intersect. Thus, the restriction of the map $f$ to $\left\{\left(u_{1}, u_{2}\right) \mid u_{1} \neq 0, u_{2} \in \mathbb{R}\right\}$ (the complement of $u_{2}$-axis in the $\left(u_{1}, u_{2}\right)$ plane) is one-to-one and onto itself. Therefore, the image of the interior of $\mathbf{R}$ is completely wrapped by the image of the boundary of $\mathbf{R}$.

Consequently, when $d=1$, in order to see if the map $f$ becomes a horseshoe map in $\mathbf{R}$, we just need to work on the dynamics of the boundaries of $\mathbf{R}$ without concerning the interior of $\mathbf{R}$ and its image. The images of the horizontal boundaries $R_{1}$ and $R_{3}$ intersect at the point $(c, e)$ as seen above, and hence, it suffices to show that

(1) the four corners of $\mathbf{R}$ move by $f$ to the LHS of $R_{4}$,

(2) the intersection $(c, e)$ is located in the RHS of $R_{2}$, and

(3) neither $f\left(R_{1}\right)$ nor $f\left(R_{3}\right)$ has any intersection with $R_{1}$ and $R_{3}$.

Set the parameters $a=4.5, b=0.85, c=0.75, d=1$, and $e=0.6$, and let $\mathbf{R}=[-0.8,0.7] \times[0.2,2.9]$. By direct calculations, it is easily confirmed that these 


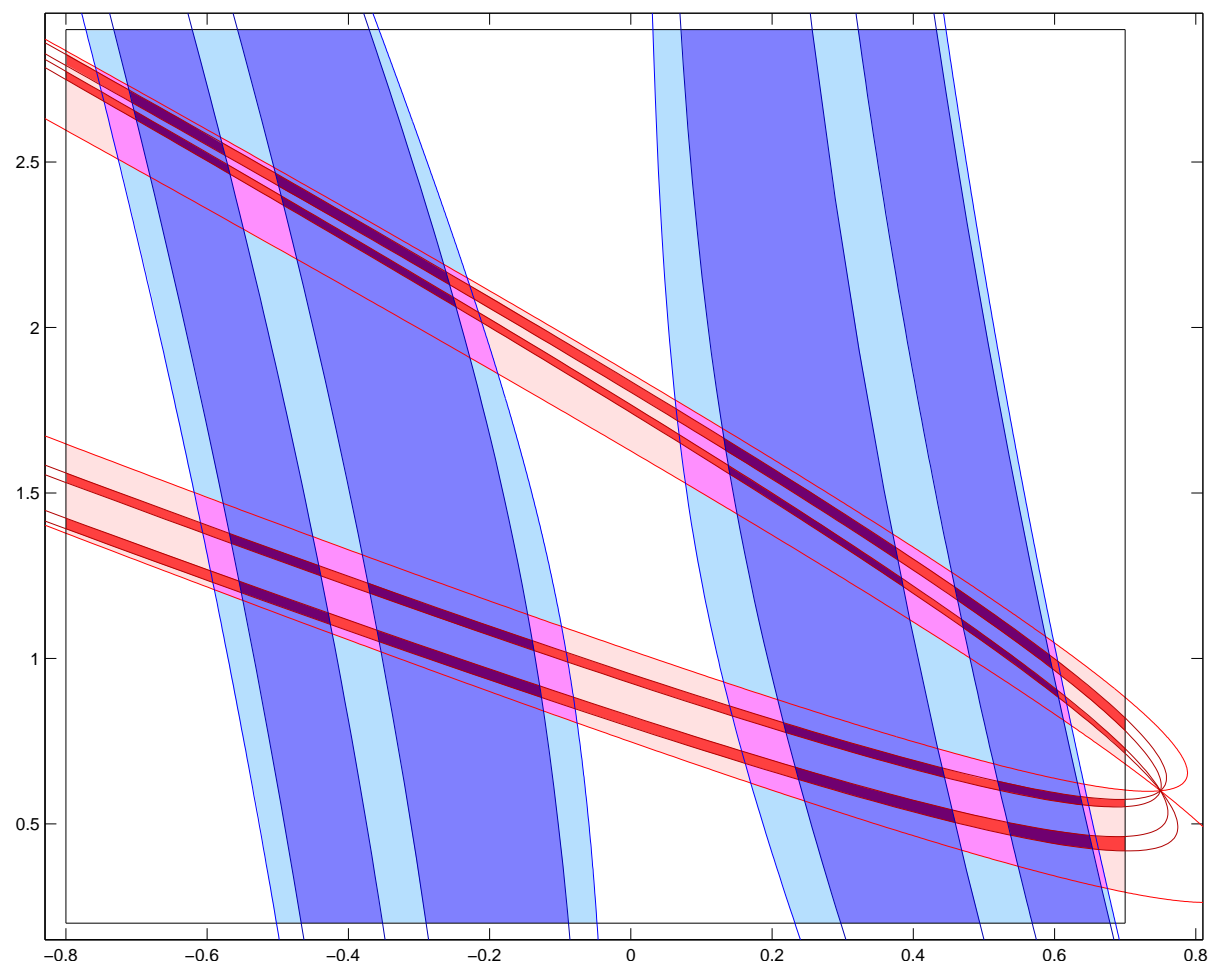

FiguRE 7. The darkest spots are the components of $f^{-2}(\mathbf{R}) \cap$ $f^{-1}(\mathbf{R}) \cap \mathbf{R} \cap f(\mathbf{R}) \cap f^{2}(\mathbf{R})$. This shows how a locally maximal hyperbolic set $\Lambda$ are created.

values for the parameters and the corners of $\mathbf{R}$ satisfies the condition above. Let

$$
\begin{aligned}
& \mathbf{R}_{1}^{h}=\left\{\left(u_{1}, u_{2}\right) \in \mathbf{R} \mid u_{1} \leq 0\right\}, \\
& \mathbf{R}_{2}^{h}=\left\{\left(u_{1}, u_{2}\right) \in \mathbf{R} \mid u_{1} \geq 0\right\} .
\end{aligned}
$$

Then $f\left(\mathbf{R}_{1}^{h}\right) \cap \mathbf{R}$ and $f\left(\mathbf{R}_{2}^{h}\right) \cap \mathbf{R}$ are disjoint and they form the two horizontal components of $f(\mathbf{R}) \cap \mathbf{R}$. Similarly, let

$$
\begin{aligned}
& \mathbf{R}_{1}^{v}=\left\{\left(u_{1}, u_{2}\right) \in \mathbf{R} \mid u_{1}+u_{2} \leq c+e\right\}, \\
& \mathbf{R}_{2}^{v}=\left\{\left(u_{1}, u_{2}\right) \in \mathbf{R} \mid u_{1}+u_{2} \geq c+e\right\} .
\end{aligned}
$$

Then $f^{-1}\left(\mathbf{R}_{1}^{v}\right) \cap \mathbf{R}$ and $f^{-1}\left(\mathbf{R}_{2}^{v}\right) \cap \mathbf{R}$ are disjoint and they form the two vertical components of $f^{-1}(\mathbf{R}) \cap \mathbf{R}$. In Figure 6 , we see how $\mathbf{R}_{i}^{h}, i=1,2$, and $\mathbf{R}_{j}^{v}, j=1,2$, move under $f$ and $f^{-1}$, respectively. Moreover, in Figure 7, the forward image and the backward image of $\mathbf{R}$ under $f$ are combined to see how the locally maximal invariant set $\Lambda$ are formulated.

Finally, one can find a constant $\lambda$ satisfying Proposition 3.8 to show that the set $\Lambda$ is hyperbolic. Instead of using the estimates for $\lambda_{1}$ and $\lambda_{2}$ in the proof of Proposition 3.8, one can use the inequalities (3.11) and (3.12) directly to find more precise constants, that is, $\lambda_{1}=1.053$ and $\lambda_{2}=1.35$, so that $\lambda=1.05$ is given for the hyperbolicity of $\Lambda$. 


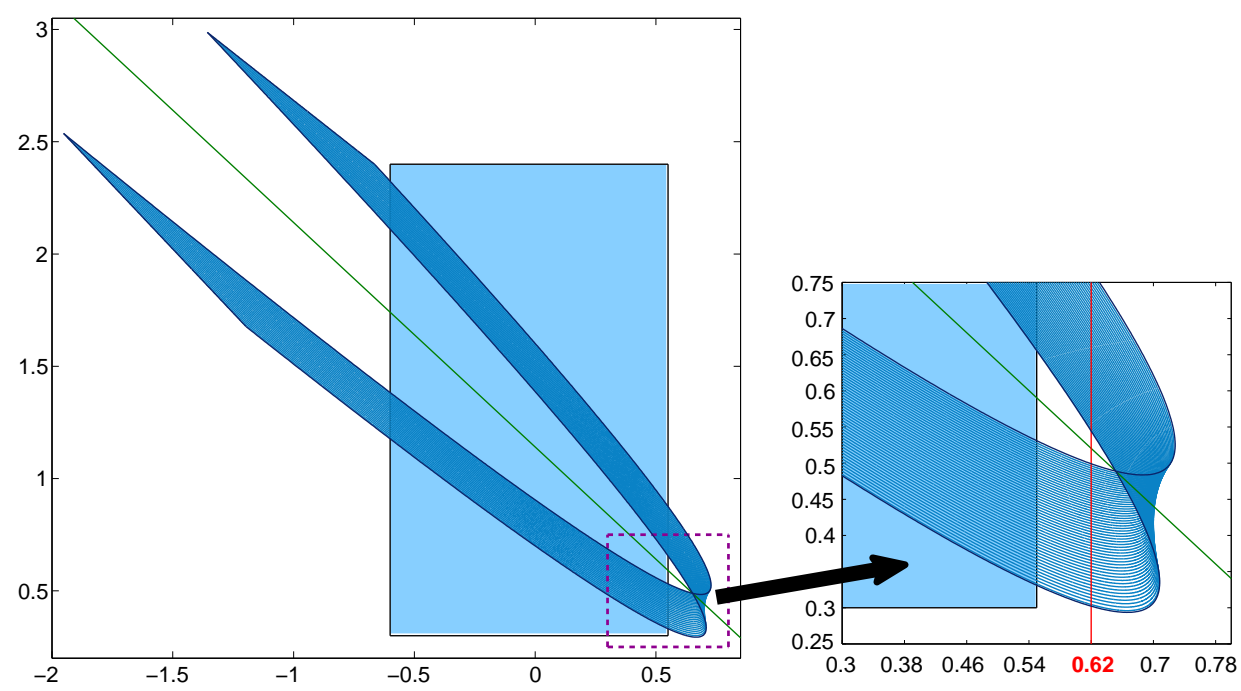

Figure 8. The image of $\mathbf{R}$ by $f$ at $a=6, b=0.6, c=0.7$, $d=1.05$, and $e=0.5$. It shows that $f(\mathbf{R}) \cap \mathbf{R}$ is composed of two disjoint horizontal connected components, so that the Smalehorseshoe structure appears. In the zoom-in region of the rectangular box, it is observed that the two horizontal components are disjoint in $[-0.7,0.62]$, at least.

4.2. $d \approx 1, d \neq 1$. For the other example, we set the parameters $a=6, b=0.6, c=$ $0.7, d=1.05$ and $e=0.5$, and the rectangular region $\mathbf{R}=[-0.6,0.55] \times[0.3,2.4]$. As shown in Section 4.1, it can be shown that all the properties concerning boundaries of $\mathbf{R}$, described in Section 3.3, hold.

However, since the restriction of $f$ to $\left\{\left(u_{1}, u_{2}\right) \mid u_{1} \neq 0, u_{2} \in \mathbb{R}\right\}$ is not one-toone correspondence when $d \neq 1$, it may not be guaranteed that the images of the boundaries of $\mathbf{R}$ wrap completely the image of the interior of $\mathbf{R}$. So, it is necessary to check the statement of Lemma 3.7. As seen in Figure 8, the image of an arbitrary horizontal line segments in $\mathbf{R}$ and the line with slope of -1 passing through the intersection of $f\left(R_{2}\right)$ with $f\left(R_{4}\right)$ have only one intersection whose $u_{1}$-coordinate is greater than 0.62 , and thus, this intersection is located in the LHS of $R_{2}$. See . Therefore, the image of the interior of $\mathbf{R}$ make two disjoint horizontal strips in $\mathbf{R}$. Similarly, one can find two disjoint vertical components in $f^{-1}(\mathbf{R}) \cap \mathbf{R}$.

\section{Conclusion}

This paper studies the CML corresponding to Turing model of morphogenesis and presents a rigorous proof for spatio-temporal chaos of this system by showing that the local map of CML has a locally maximal hyperbolic set. This work is based on the result of Afraimovich and Pesin [1], which provides a set of sufficient conditions of invariant measures for guaranteeing spatio-temporal chaos in lattice systems. We also perform the numerical computations for hyperbolic properties of this system. 


\section{REFERENCES}

[1] V.S. Afraimovich and Ya. Pesin, Travelling Waves in Lattice Models of Multi-dimensional and Multi-component Media : I. General Hyperbolic Properties, Nonlinearity, 6 (1993), 429455.

[2] V.M. Alekseev, Quasirandom Dynamical Systems. I. Quasirandom Diffeomorphisms, Math. USSR Sb., 5 (1968), 73-128.

[3] L.A. Bunimovich and Ya. Sinai Space-Time Chaos in Coupled Map Lattices, Nonlinearity, 1 (1988), 491-516.

[4] R. Devaney and Z. Nitecki, Shift automorphism in the Hénon mapping, Comm. Math. Phys., 67 (1979), 137-148.

[5] A. Gierer and H. Meinhardt, A Theory of Biological Pattern Formation, Kybernetik, 12 (1972), 30-39.

[6] M. Jiang and Ya. Pesin, Equilibrium Measures for Coupled Map Lattices: Existence, Uniqueness, and Finite-Dimensional Approximations, Comm. Math. Phys., 193:3 (1998), 675-711.

[7] K. Kaneko, Theory and Applications of Coupled Map Lattices, Wiley, New York (1993).

[8] H. Kang, Dynamics of Local Map of a Discrete Brusselator Model: Eventually Trapping Regions and Strange Attractors, DCDS-A, 20:4 (2008), 939-959.

[9] L. Ji and Q. Li, Effect of spatiotemporal perturbation on Turing pattern formation, Phys. Let. A, 329, (2004), 309-317.

[10] K. Maginu, Reaction-Diffusion Equation Describing Morphogenesis, Mathematical Bioscience, 27 (1975), 17-98.

[11] P. Maini, Spatial and Spatiotemporal Pattern Formation in Generalised Turing Systems, Math. Comput. Model., 32:11 (1996), 71-77.

[12] J. Moser, Stable and Random Motions in Dynamical Systems, Princeton University Press, Princeton, (1973).

[13] D. Orendovici and Ya. Pesin, Chaos in Traveling Waves of Lattice Systems of Unbounded Media, IMA Vol. Math. Appl., 119, Springer, (2000), 327-358.

[14] Ya. Pesin and Ya. Sinai Space-Time Chaos in Chains of Weakly Hyperbolic Mappings, Advances in Soviet Math., 3 (1991), 165-198.

[15] D. Tanaka, Turing Instability Leads Oscillatory Systems to Spatiotemporal Chaos, Prog. Theor. Phys. Suppl., 161 (2006), 119-126.

[16] A. Turing, The Chemical Basis of Morphogenesis, Philosophical Transactions of the Royal Society of London, 237 (1952), 37-72.

Department of Mathematics, Hokkaido University, Sapporo 060-0810, JAPAN

E-mail address: kang@math.sci.hokudai.ac.jp 